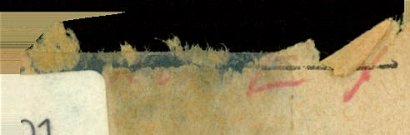

\title{
SOUND ABSORPTION BY POROUS MATERIALS I
}

\author{
by J. v. D. EIJK, C. W. KOSTEN and W. KOK
}

Geluidscommissie T.N.O. and Laboratorium voor Technische Physica der Technische Hogeschool, Delft

\section{Sull. mi Gehidhaummisni T.N.D.}




\section{Summary}

It is a well-known fact that, in order to obtain maximum absorption, sound-absorbing materials must not be fastened directly to the structural wall or ceiling, but must be kept at a certain distance, small in practice, from the wall or the ceiling. After describing a new interferometer some results concerning the influence of the thickness of the layer of air between material and wall are given. These results are discussed and explained theoretically. Most remarkable is the large influence of very thin layers of air on the frequency at which a maximum absorption is obtained.

$\S 1$ Introduction. Under the same title a series of five papers were published in Physica ${ }^{1-5}$ ). This paper may be considered as a continuation of that serjes. It is hardly possible and it seems also unnecessary to give here a complete survey of the contents of the previous articles. A few words will suffice perhaps to give some idea of what problems were dealt with. Sound absorbing materials are wanted in practice for noise abatement in offices, factories and large rooms, to shorten the reverberation time as well as to eliminate echoes in concert halls and the like. Porous materials are the most widely used absorbers in practice. Their absorption coëfficient $a$ (absorbed energy divided by incident energy) depends upon frequen$c y$, the thickness of the absorbing layer and certain partly known constants of the material. It would be a great advantage, and therefore has been the object of many papers, if it were possible to compute $a$ from these variables. In these calculations the porosity $h$ and the specific air resistance $\sigma$ mostly enter as easily measurable quantities $\left.{ }^{6-10}\right)$. It was found experimentally that in porous materials the wave length (and the velocity of sound) are considerably smaller 
than in free air. To account for these facts, the introduction of a third constant turned out to be necessary ${ }^{2}$ ), the so-called structure factor $k$, which is greater than one if there are pores in other directions than perpendicular to the wall surface or if there are pores of which the cross-section varies appreciably along the length of the pores (which, of course, is always the case in practice). $k$ is a measure of the extent to which these deviations from an ideal ",honeycomb" material are present. See also Korringa, Kronig and $\mathrm{S} \mathrm{m} \mathrm{it}{ }^{11}$ ), where an exact theoretical treatment of such a case is given, that leads to results, which to some extent can also be obtained with a $k>1$. The wave length and the velocity of sound are proportional to $\sqrt{ } k$, which may be looked upon as the definition of $k$. The numerical value of $k$ in ordinary cases lies between 1 and 10.

Experimental results are mostly given in terms of the specific acoustical impedance $z$ (sound pressure over normal particle velocity at the surface of the absorbing layer). This makes a comparison with theory very simple, since theoretical results can easily be given in the form of impedances, whereas, when $z$ is known, $a$ can be obtained in a direct way by using the so-called circle diagram, connecting $a$ and the phase jump $\Delta$ between incident and reflected sound wave at the reflecting surface with the complex impedance. So when $z$ is given, the figure $a$ that interests the practical man directly can be read from a graph. More information concerning this important graph may be found in a paper by K o st en in this issue (page 38).

For reasons of simplicity only absorption in a direction perpendicular to the absorbing surface is considered. The measurements can be easily carried out in this case in a so-called interferometer, which consists of a tube, closed at one end with the absorbing sample under test, at the other end of the tube by a loudspeaker. The latter emits a sound wave towards the sample where it is reflected, perhaps with a decreased amplitude owing to absorption. At regular intervals of one quarter of a wave length we observe maxima and minima of sound pressure, the minima being exactly zero only when absolutely no absorption takes place. Absorption by the side walls is avoided as far as possible. So the absorption coëfficient of the sample can be computed from the ratio between maximum and minimum sound pressure in the tube. This ratio is measured directly in practice. Also the distance between the place of minimum sound pressure and 
the surface of the sample can be measured, from which distance, measured in wave lengths, the phase difference between the sound pressure of the incident and reflected wave at the surface can be computed. With the aid of $a$ and $\Delta$ the impedance $z$ at the surface can be determined in the complex impedance diagram.

§ 2. Description of a nere interferometer. In principle practically all interferometers for sound at normal incidence are the same. They differ only in the way in which the sound pressure in the interference pattern is measured. In large tubes (which are used with low frequencies) the sound pressure is sometimes measured directly by a small microphone in the sound field. At higher frequencies (narrower interferometer tubes) this method causes too much disturbance of the sound field. Then a so-called exploring tube may be used, a tube of very small diameter in comparison with that of the interferometer, forming an acoustical connection between the microphone outside the tube and the measuring point inside. In order to avoid even the slight disturbance due to this exploring tube another method was followed up to now in our laboratory ${ }^{12}$ ). Along the same lines a new interferometer was constructed, in which some shortcomings of the older one were avoided (see fig. 1). The whole cylindrical interfero-

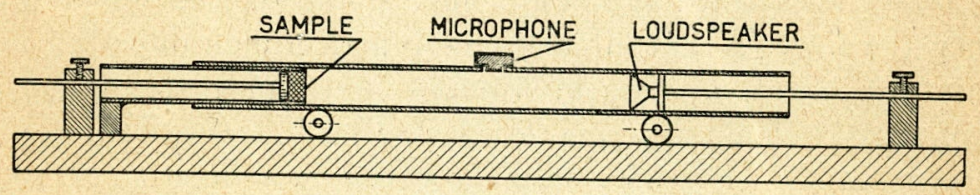

Fig. 1. Acoustical interferometer.

meter tube of brass $3.5 \mathrm{~mm}$ thick, may be moved relative to the loudspeaker and the sample holder which fit in an airtight way, like pistons, into the tube and are rigidly fixed to the heavy iron support. The tube can be moved over about $43 \mathrm{~cm}$. The microphone is mounted inside in the tube wall. It consists of a stiff membrane (high impedance), lying in the "plane” of the inner surface, with a square Rochelle salt cristal mounted behind, one corner of which is brought into contact with the membrane by means of a light rod.

The surface of the membrane is about $1 \mathrm{~cm}^{2}$. This might seem a source of errors, since it will be impossible to measure the sound 
pressure at one "single" point. This objection, however, is not correct. At places where the sound pressure is a maximum, the pressure varies very slowly with the displacement (maxima are very flat), so that hereby no difficulties are to be expected. The minima are very sharp indeed; denoting the distance from a minimum by $x$, the sound pressure may be written

$$
p_{i_{\text {max }}} \cos \omega\left(t-\frac{x}{c}\right)-p_{r_{\text {max }}} \cos \omega\left(t+\frac{x}{c}\right)
$$

where $p_{i_{\max }}=$ amplitude of sound pressure of incident wave,

$$
\begin{aligned}
p_{r_{\max }} & =\text { dito of reflected wave, } \\
c & =\text { velocity of sound, }
\end{aligned}
$$

hence

$$
\left(p_{i_{\text {max }}}-p_{r_{\text {max }}}\right) \cos \omega t \cos \frac{\omega x}{c}+\left(p_{i_{\text {max }}}+p_{r_{\text {max }}}\right) \sin \omega t \sin \frac{\omega x}{c}
$$

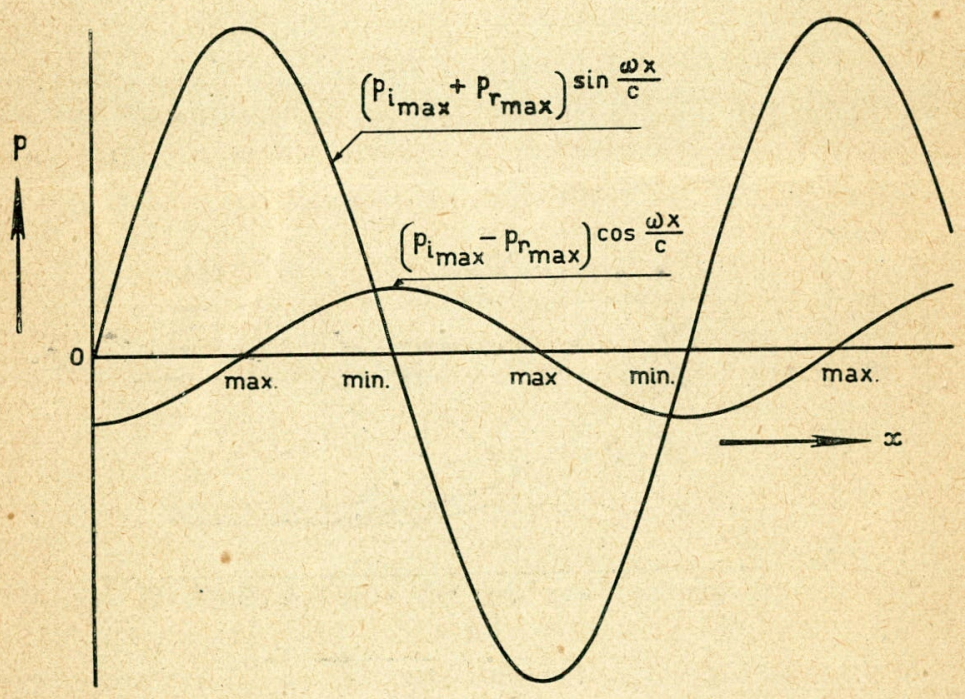

Fig. 2. The interference pattern can be considered as the superposition of two standing waves (timefunctions orthogonal however!). At the minima the grdater standing wave is zero and symmetrical with respect to the minimum. A large microphone, therefore, may be used to explore the sharp minima.

The pressure at the minimum is the pressure at $x=0$, i.e. the first term, that varies with $\cos \omega x / c$, which has a flat maximum at 
the minimum under test. The second term, varying with $\sin \omega x / c$ does not in the least contribute to the minimum. This term is responsible for the sharpness of the minimum. The average value of this term between $-x$ and $+x$ however is zero, so the force on the membrane, when it is placed symmetrically with respect to the minimum, is proportional to the pressure to be measured. In fig. 2 the situation is shown in a graph. We can see at once from fig. 2 that there is no principal difference between the situation at maxima or minima. Since the dimensions of the membrane are not negligible in comparison with the wave length, the mean value of $\cos \omega x / c$ over the membrane is smaller than unity. This, however, is the case equally for minima and maxima so that the ratio of sound pressures is always measured in a correct way, independent of the size of the microphone membrane.

Finally, a practical feature of the sample holder is to be noticed. A rigid brass plate of $10 \mathrm{~mm}$ thickness is situated at the back of the sample. This plate may be moved backwards over about $16 \mathrm{~cm}$, so it is very easy to carry out investigations on sound absorption with samples, backed by an adjustable layer of air, without touching the sample itself.

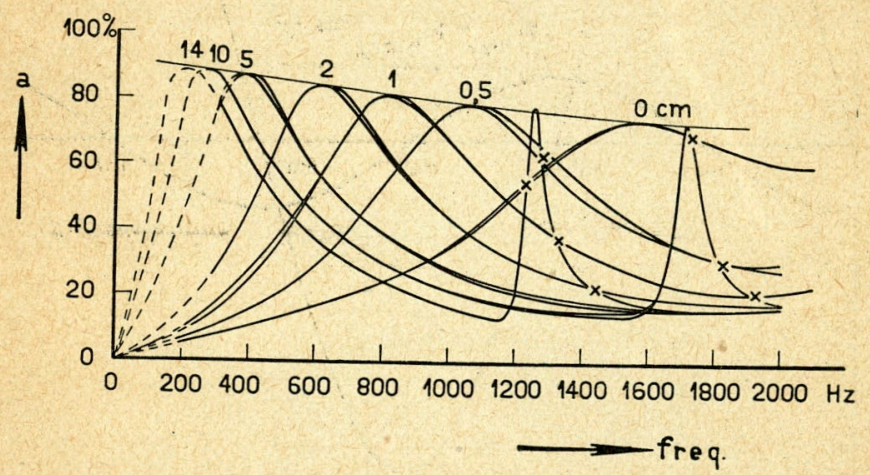

Fig. 3. Absorption coëfficient as a function of frequency for different layers of air at the back of the sample. The thickness of the layer of air is given in $\mathrm{cm}$ at each curve. Notice the large influence of very thin layers of air!

§3. Sound absorption by a sample of acoustic plaster, backed with different layers of air. Fig. 3 shows results of sound absorption measurements, carried out on an acoustic plaster (see fig. 4) of $21 \mathrm{~mm}$ 
thickness, consisting of granular pumice ( $1-4 \mathrm{~mm}$ diameter), cemented together with a small amount of anorganic adhesive and thus giving a material with rather wide pores. The specific air

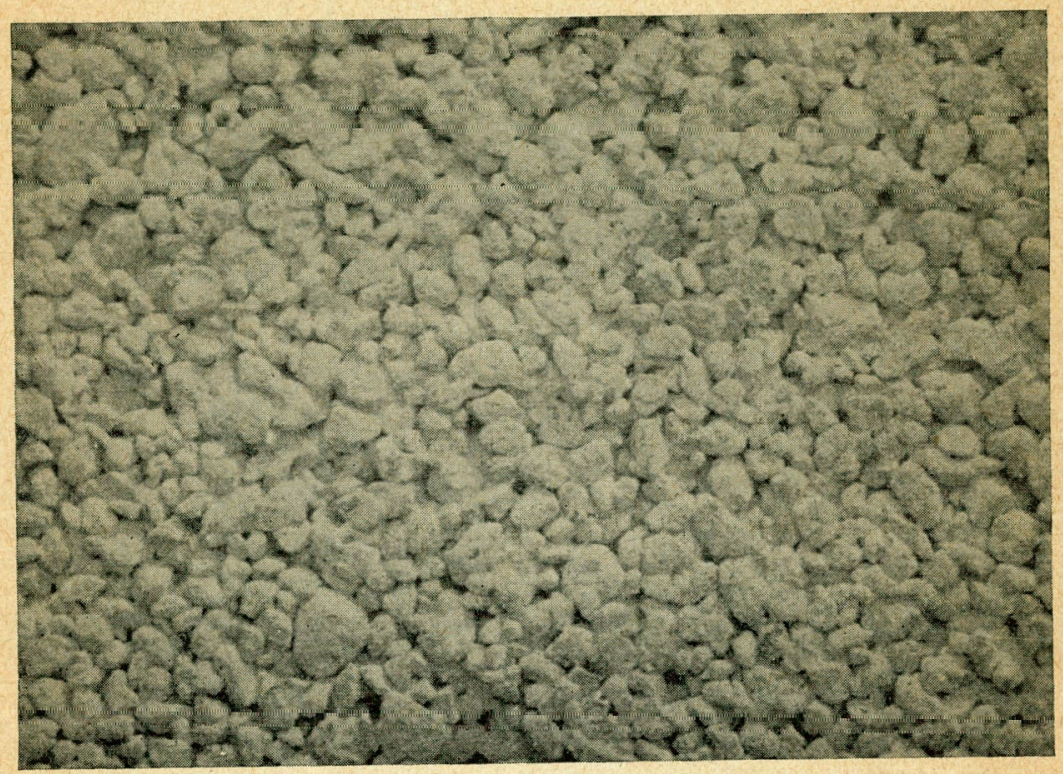

Fig. 4. Photograph of the sample, in 2-fold magnification.

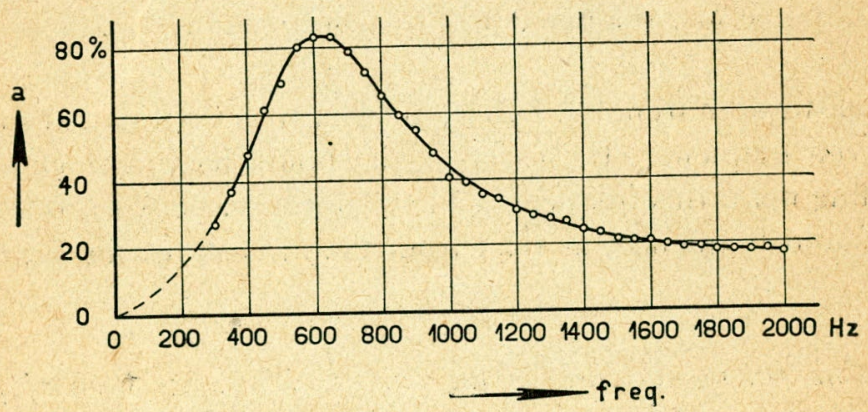

Fig. 5. The curve for $2 \mathrm{~cm}$ air of fig. 3 with measuring points added.

resistance (static value turned out to be $31.10^{3} \mathrm{~N} \mathrm{sec} / \mathrm{m}^{4}$ (31 dyne $\left.\mathrm{sec} / \mathrm{cm}^{4}\right)$, the porosity $72 \%$. Each curve was measured twice, which explains why at several places the curves are doubled.

At a first glance at fig. 3 the large influence of very thin layers of 
air seems most remarkable. Whereas without a layer of air maximum absorption occurs at $1570 \mathrm{~Hz}, 5 \mathrm{~mm}$ of air suffice to shift this maximum to about $1070 \mathrm{~Hz}, 20 \mathrm{~mm}$ to as low as $600 \mathrm{~Hz}$. No measuring points are given in fig. 3 in order not to overload it. To give an idea of the magnitude of unsystematic errors, fig. 5 shows the curve for $20 \mathrm{~mm}$ air with measuring points.

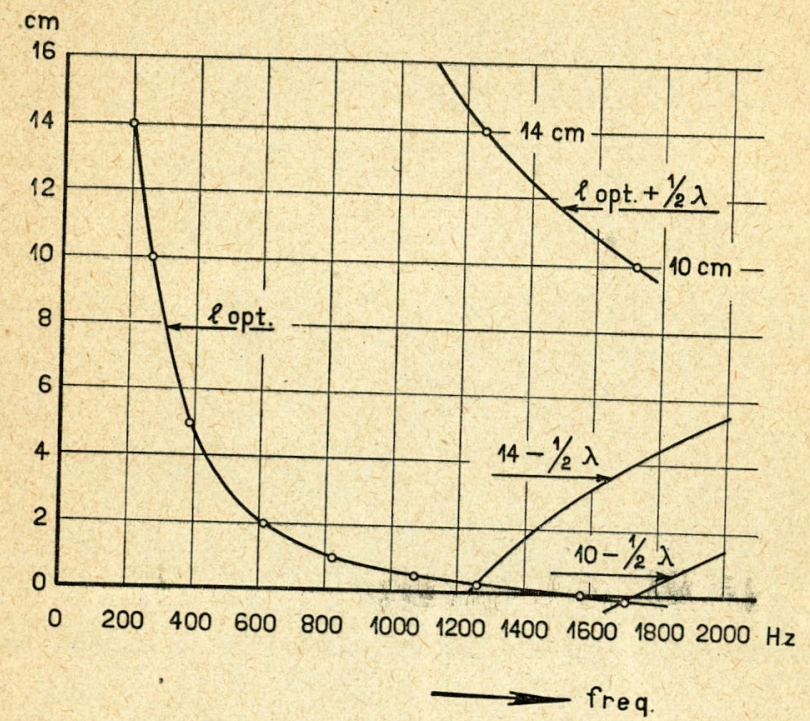

Fig. 6. The thickness of the layer of air, at which maximum absorption occurs, as a function of frequency (left curve).

It is easily seen from fig. 3 that, in order to get optimal absorption at a given frequency, the layer of air should have a thickness $l_{\text {opt }}$ depending upon this frequency. The connection between this thickness $l_{\text {opt }}$ and frequency, as read from fig. 3 , is shown in fig. 6 (left curve).

\$4. The interconnection of the curves of fig. 3 ; check of figure. It is quite easy to check the curves of fig. 3 at certain points. The (acoustical) impedance of a layer of air of one half wavelength thickness $(\lambda / 2)$, backed with a rigid plate, is infinite, so the absorption coëfficient $a$ of any sample must be the same whether it is backed direct by a rigid plate or by that plate at a distance $\lambda / 2$ (or even in general $n \lambda / 2)$ from the back of the sample. In mathematical terms: 
the absorption coëfficient $a$ of a given sample is a function of the frequency and the thickness of the layer of air. As to the dependence upon this thickness $l$ we know that $a$ must be a periodic function with periode $\lambda / 2$, i.e.

$$
a(l)=a(l+n \lambda / 2) \text { at constant frequency. }
$$

Take for instance

$$
l=0 \text {, }
$$

$l+\lambda / 2=14 \mathrm{~cm}$ (from which there results the frequency $\nu=1232 \mathrm{~Hz}$ ), then we conclude that at $1232 \mathrm{~Hz}$ a for the sample without layer of air must be equal to $a$ for the sample backed with $14 \mathrm{~cm}$ layer of air, i.e. the curves for $l=0$ and $l=14 \mathrm{~cm}$ must have a point of intersection a $1232 \mathrm{~Hz}$. This, indeed is the case within experimental error. Also if

$$
\begin{gathered}
l=0.5 \mathrm{~cm}, \\
l+\lambda / 2=14 \mathrm{~cm}(\text { from which } v=1278 \mathrm{~Hz}),
\end{gathered}
$$

the point of intersection of the curves for $l=0.5$ and $\mathrm{cm} l=14 \mathrm{~cm}$ turns out to be at $1278 \mathrm{~Hz}$, in accordance with the measurements. To have a check at the top of the second resonance peak in the 14 cm-curve we take

$$
\begin{gathered}
l=0.3 \mathrm{~cm} \\
l+\lambda / 2=14 \mathrm{~cm}(\text { therefore } v=1259 \mathrm{~Hz}) .
\end{gathered}
$$

The $0.3 \mathrm{~cm}$ - and $14 \mathrm{~cm}$-curve will have a point of intersection quite near to the envelope; therefore $1259 \mathrm{~Hz}$ must be a good approximation to the second resonance frequency of the $14 \mathrm{~cm}$-curves. This again agrees with experiment.

In this way we computed the seven points of the second resonance peaks in the $10 \mathrm{~cm}$ - and $14 \mathrm{~cm}$-curves, marked with a cross in fig. 3. The agreement with experiment is evident. For these calculations the auxiliary curves to the right in fig. 6 may be of some use. They can serve e.g. to calculate with higher precision the position of the two second resonance peaks.

\$5. Theoretical explanation of the large influence of thin layers of air. In the last $\S$ the results of fig. 3 were found to be in good agreement with each other as far as could be verified. We now proceed to give a 
simple theory that enables us to predict at which frequency maximum absorption occurs. For this purpose we first neglect dissipation, since on the one hand the damping effects are rather small in our case, whereas their influence on resonance frequencies, that turn out to stand in close connection with the frequencies of maximum absorption, is only of second order. Without dissipation it is quite easy to calculate the (purely imaginary) impedance of the sample considered. Those frequencies for which the impedance becomes zero are called resonance frequencies. The effect of small dissipation on the computed impedance will be such that to the computed, purely imaginary impedance a real part is added. Now the total impedance is no longer purely imaginary and some absorption will be the result. The effect at resonance frequencies will be very pronounced, for here the impedance zero will increase towards $\varrho c$, the real wave resistance of air. If at resonance, owing to just enough dissipation, the impedance equals exactly @c, complete absorption $(100 \%)$ occurs. In fig. 3 , therefore, abșorption peaks will be found at the frequencies at which the sample under test would have impedance zero if there were no dissipation at all.

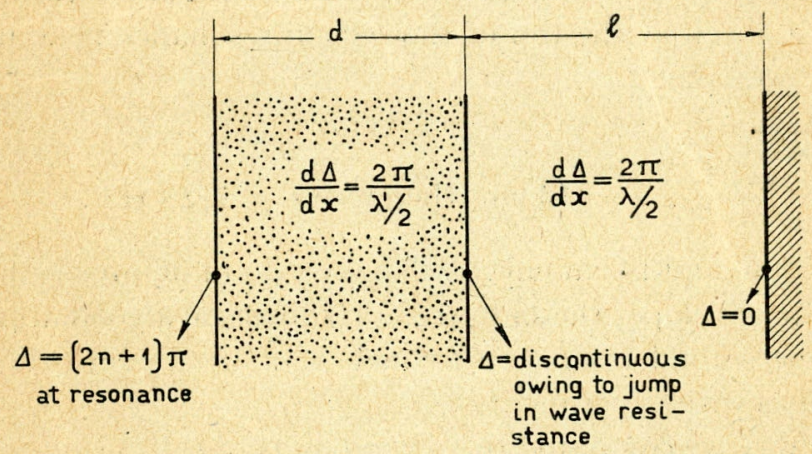

Fig. 7. Scheme indicating the general line along which the resonance frequencies of a (porous) material (thickness $d$ ) on a layer of air (thickness l) may be computed.

Fig. 7 indicates the way in which these resonance frequencies can be established. The standing wave in the layer of air can be considered as the superposition of an incident wave and a reflected one of equal strength (no absorption at the wall). The phase difference $\Delta$ of the sound pressures of both waves at the wall is zero (pressures are doubled, velocities compensated). Proceeding from the wall into 
the layer of air, the phase difference varies proportional to the displacement, $2 \pi$ radians for each half wave length; therefore at a distance $l$ from the wall, just at the boundary of the material but still in the layer of air, $\Delta$ amounts to

$$
\Delta_{l, a i r}=4 \pi l / \lambda .
$$

On passing the boundary of the material, $\Delta$ changes discontinuously owing to the sudden change in wave resistance. If the impedance in the material at a point with coördinate $x$ be defined as the ratio of $p$ and the average value of $v$, the average being taken over the whole plane $x=$ constant, solid matter included, there is no discontinuity at the boundary as to the impedance, for $p$ as well as the average velocity are continuous at the boundary. $\Delta$ depends, however, (see below) upon the ratio of impedance and wave resistance, and this ratio is actually discontinuous, owing to the discontinuity in wave resistance. In air the wave resistance amounts to $\varrho c$, in the material to $\varrho c \sqrt{k} / h$, where $h=$ porosity and $k=$ structure factor ${ }^{2}$ ). Now there is a very simple relation between a purely imaginary impedance $z$, the real wave resistance $z_{w}$ and the phase difference $\Delta$, namely

$$
z=j z_{w} \operatorname{ctg} \frac{1}{2} \Delta
$$

which may be deduced either from the well-known relation

$$
\text { complex reflection coëff. } r=\frac{p_{r}}{p_{i}}=|r| \exp \cdot j \Delta=\frac{z-z_{w}}{z+z_{w}}
$$

or from the circle diagram, giving in the complex $z$-plane circles of constant $\Delta$ and absorption $a$ (see page 39). Now, since $z$ is continuous, the discontinuity in $\Delta$ may be computed at once from the equation

$$
z_{w \text { mat }} \operatorname{ctg} \frac{1}{2} \Delta_{l, \text { mat }}=z_{w \text { air }} \operatorname{ctg} \frac{1}{2} \Delta_{l, \text { air }}
$$

or

$$
\Delta_{l, \text { mat }}=2 \operatorname{arcctg}\left[\frac{h}{\sqrt{ } k} \operatorname{ctg} \frac{1}{2} \Delta_{l, a i r}\right] .
$$

E.g. taking $h / \sqrt{k}=0.1$ and $\Delta_{l \text {, air }}=90^{\circ}$, we find

$$
\Delta_{l, \text { mat }}=168^{\circ} \text {, a discontinuity of about } 80^{\circ} \text { ! }
$$

The change of the phase difference on proceeding over the distance 
$d$ through the material can at once be given as $4 \pi d / \lambda^{\prime}$, analogous to that in the layer of air. It should be noticed, however, that this time $\lambda^{\prime}$ enters, i.e. the wave length in the material $\left.\left(\lambda^{\prime}=\lambda / \sqrt{k}^{2}\right)\right)$. Therefore we find for the total phase difference at a distance $l+d$ from the wall, however just inside the material,

$$
\Delta_{\text {tot }}=\frac{4 \pi d}{\lambda^{\prime}}+2 \operatorname{arcctg}\left(\frac{h}{\sqrt{k}} \operatorname{ctg} \frac{2 \pi l}{\lambda}\right) .
$$

At resonance $\Delta_{t o t}$ must be equal to $\pi, 3 \pi, 5 \pi, \ldots$ etc., for this being so, the sound pressure of incident and reflected wave at the surface of the sample will compensate each other and the impedance will be zero. This leads to the following transcendental equation for the resonance frequencies:

$$
\operatorname{tg} \frac{2 \pi v l}{c} \operatorname{tg} \frac{2 \pi v d \sqrt{k}}{c}=\frac{h}{\sqrt{k}} .
$$

$d$ has in our case a fixed value of $2.1 \mathrm{~cm}, c$ is $34500 \mathrm{~cm} / \mathrm{sec}, h=0.72$. If $k$ were known, we could compute $v_{\text {res }}$ as a function of $l$ (for $n=1$ leading to the first resonance frequency and so on). We must, however, deduce $k$ from experiment, for which purpose we can use the experimental fact that for $l=0$ the first resonance frequency is about $1570 \mathrm{~Hz}$. This leads to $k=6.85$. Now we are able to compute at each frequency the thickness of the layer of air that is necessary for resonance, i.e. the left curve of fig. 6 must be found. The general trend of the curve turns out to be correct; however, at low frequencies too high values for $l$ are computed, higher than could be caused by measuring errors. Assuming a smaller $h$ than 0.72 gives closer agreement. In fig. 8 some results of calculations with different $h$-values (curves b, c and d for resp. $h$-values of $0.28,0.39$ and 0.52 ) are given together with the experimental curve a (left curve of fig. 6 , reproduced here to facilitate comparison). The curve c $(h=0.39)$ agrees fairly well with experiment. The discrepancies at low frequencies might be due to erroneous extrapolation, for in reality no absorption peaks below $270 \mathrm{~Hz}$ have been measured (see the dotted parts of the curves of fig. 3 at low frequencies). The discrepancy between the static value $h=0.72$ and the dynamic value $h=0.39$ is rather great. The only reasonable explanation seems to be that in $h=0.72$ also those cavities are included that are hardly in connection with the outer air and have no function at all at audiofrequencies. 
In more mathematical terms: those cavities of which the time constant ("the $R C$ - or relaxationtime") is large in comparison with the period of the sound do not take part in the dynamical process.

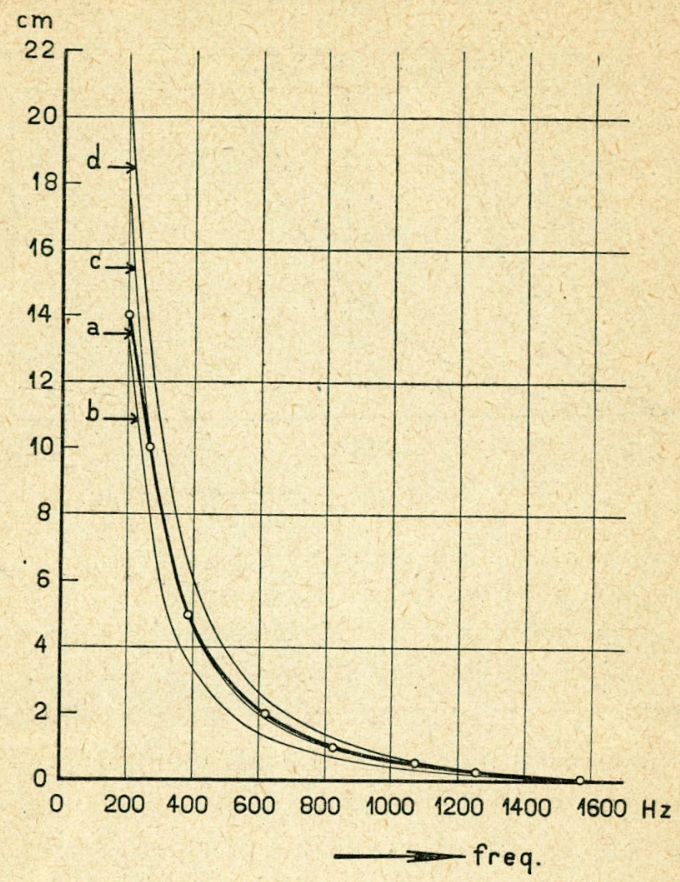

Fig. 8. Experimental curve of fig. 6, compared with theoretical curves.

The question may be raised which material constants are of primary importance for the large effect of thin layers of air on the resonance frequency. Fig. 9 gives an answer to this question for the particular case of a sample of $2.1 \mathrm{~cm}$ thickness. Six curves are given, representing the first resonance frequency as a function of the three variables $h$ (porosity), $k$ (structure factor) and $l$ (thickness of layer of air). When $l=0$ (no layer of air) this frequency does not depend upon $h$, the "curves" being straight lines, depending upon the structure factor only. When $l=1 \mathrm{~cm}$, the resonance frequency depends upon $h$ and $k$. The influence of the layer of air may be measured in the relative drop in resonance frequency caused by the introduction of this layer. It is read directly from the graph that the resonance frequency drop with $k=4$ and $h=0.8$ amounts to 2060 
$1340=720 \mathrm{~Hz}$ (about $35 \%$ ), whereas with $k=4$ and $h=0.1$ it is increased to $2060-590=1470 \mathrm{~Hz}$ (about $71 \%$ ). The influence of variations in $k$ is simple, all frequencies being approximately proportional to $\sqrt{ } k$. Increasing $k$ from 1 to 9 therefore decreases the

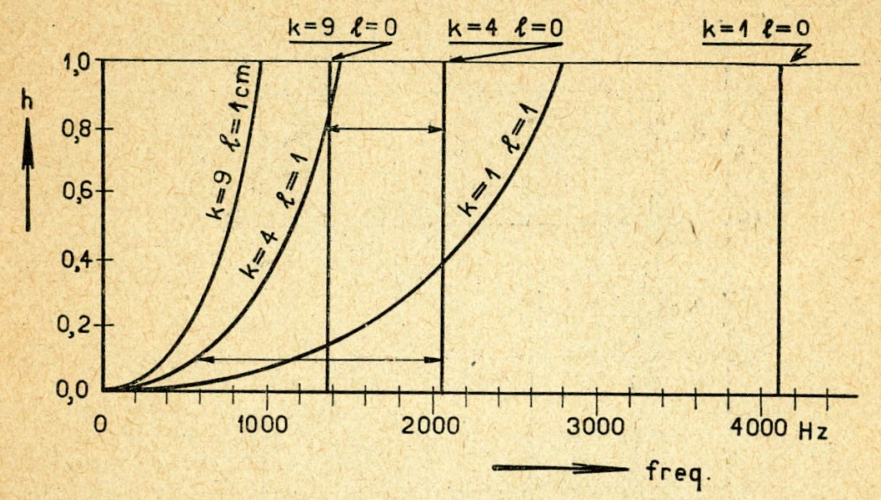

Fig. 9. Graph showing the influence of $1 \mathrm{~cm}$ layer of air on the resonance frequency (frequency of maximum absorption) as a function of the porosity $h$ and the structurefactor $k$. Thickness of sample $2,1 \mathrm{~cm}$.

resonance frequency three times. Such large variations, however, are seldom obtained, all normal materials yielding. $k$-values of about 6-8. Decreasing $h$ may therefore be looked upon as an adequate means of lowering the resonance frequencies, provided a layer of air is present and relatively low $h$-values are used.

Received 1st May 1947.

\section{REFERENCES}

1) J. va n den Eijk and C. Zwikker, Physica 8, 149, 1941.

2) C. Z w i k ker, J. van den E ij k and C. W. Kost e n, Physica 8, 469, 1941

3) C. Z wikker, J. v a n den E ij k and.C. W. Koste n, Physica 8, 1094, 1941.

4) C, Z w i k k e r, Physica 8, 1102, 1941.

5) C. Z wikker, J. van den E ijk and C. W. Kost e n, Physica 10, 239, 1943.

6) H. W ü s t, Hochfrequenztechn. u. Elektroakustik 44, 73, 1934.

7) V. K ü h 1 and E. M e y e r, Berl. Ber. Phys. Math. Kl. 26, 416, 1932.

8) L. Cremer, Elektr. Nachr. Techn. 10, 242, 1933.

9) L. Cre m e r, Elektr. Nachr. Techn. 12, 333, 1935.

10) A. F. M onn a, Physica 5, 129, 1938.

11) J. Korringa, R. Kronig and A. Smit, Physica 11, 209, 1945.

12) C. W. Koste $\mathrm{n}$ and C. Zwikker, Physica 8, 933, 1941. 

